\title{
Improving Semantic Parsing Using Statistical Word Sense Disambiguation (Student Abstract)
}

\author{
Ritwik Bose, Siddharth Vashishtha, James Allen \\ \{rbose, svashis3, james\}@cs.rochester.edu
}

\begin{abstract}
A Semantic Parser generates a logical form graph from an utterance where the edges are semantic roles and nodes are word senses in an ontology that supports reasoning. The generated representation attempts to capture the full meaning of the utterance. While the process of parsing works to resolve lexical ambiguity, a number of errors in the logical forms arise from incorrectly assigned word sense determinations. This is especially true in logical and rule-based semantic parsers. Although the performance of statistical word sense disambiguation methods is superior to the word sense output of semantic parser, these systems do not produce the rich role structure or a detailed semantic representation of the sentence content. In this work, we use decisions from a statistical WSD system to inform a logical semantic parser and greatly improve semantic type assignments in the resulting logical forms.
\end{abstract}

\section{Introduction}

In this work, we improve the sense disambiguation of a logical semantic parser, The TRIPS Parser (Allen, Swift, and De Beaumont 2008), by integrating advice from a statistical Word Sense Disambiguation (WSD) system, SupWSD (Papandrea, Raganato, and Bovi 2017).

We provide sense decisions from SupWSD to the TRIPS parser to augment existing heuristics and try to keep the correct sense in the search space.

Given word sense advice from, SupWSD (Papandrea, Raganato, and Bovi 2017), a statistical word sense disambiguation system, we improve the quality of logical forms produced by the TRIPS semantic parser. We provide two types of hints to the parser: Prehinting primes the parser to consider certain senses at the beginning of the parsing process while progressive hinting continually directs the parser towards certain senses throughout the process.

\section{The TRIPS Parser}

The TRIPS parser is a best-first bottom-up chart-parser with a hand-built, lexicalized context-free grammar. A core semantic lexicon produces lexical entries which unify syntactic features and templates with semantic types drawn from

Copyright (C) 2020, Association for the Advancement of Artificial Intelligence (www.aaai.org). All rights reserved. an ontology. Constituents, consisting of a word, semantic type and semantic roles, are constructed bottom-up and scored by several heuristics for likelihood. Broad coverage for lexical entries is achieved by hand-mapping the multiple inheritance WordNet 3.0 hierarchy (Fellbaum 1998) to the single inheritance TRIPS ontology.

The ontology is a hand-crafted single-inheritance hierarchy where nodes specialize or override hierarchical features and argument templates from their parent. Each sense is related to its parent and children in both syntax and semantics, so word instances which share a sense also share grammatical constructions. Each sense specifies a set of roles with type and feature restrictions. Hence, the graphical structure of logical forms generated by the parser is as much determined by the word senses provided by TRIPS types as semantic roles.

In order to leverage the broad lexical coverage provided by WordNet, the TRIPS ontology also contains manual mappings from WordNet synsets to ontology types. Using a simple subsumption algorithm, sub-trees of the WordNet hierarchy are assigned to TRIPS senses.

In this work, we use a supervised-system SupWSD (Papandrea, Raganato, and Bovi 2017) which provides a probability distribution over WordNet senses for a given sentence. The system uses an SVM classifier to predict the word senses and its features include various linguistics properties such as POS tags, syntactic relations, local collocation, word-embeddings, and information about surrounding words. We show that combining the knowledge of a statistical WSD with a semantic parser results into an improved performance of the semantic parser.

\section{Methodology}

Word sense advice consists of a word, sense, and score over a token span. On receiving a new sentence, the parser receives a message indicating SupWSD's sense preferences for each span. This contains all the information required to perform either type of hinting. The advice module converts WordNet senses output by SupWSD into a TRIPS ontology type. This is to ensure minimal interaction between the word sense advice and other internal heuristics in the parser. 


\begin{tabular}{l|cc|c|c|c}
\hline \multicolumn{1}{c}{ Metric } & SupWSD & Plain & Pre & Prog & Comb \\
\hline Accuracy & 66.19 & 39.42 & 50.33 & 42.45 & 53.19 \\
\hline WuP & 84.22 & 73.97 & 79.12 & 75.75 & 80.53 \\
\hline SemFac & 70.26 & 49.33 & 60.76 & 53.38 & 63.37 \\
\hline
\end{tabular}

Table 1: Results from evaluation on SemEval2013 (Navigli, Jurgens, and Vannella 2013). We compare accuracy, WuPalmer and semantic factor agreement.

Prehinting ensures the parser adds a lexical entry with the given sense to its chart. Prehinted senses start on the chart with a score of 1 , which generally ensures they are considered highly early in the parsing process.

Progressive Hinting is performed as constituents are expanded on the chart. At each stage, constituents are pruned from the chart performed to ensure the search space remains feasible. The goal of progressive hinting is to subtly augment the score of constituents containing senses advised by SupWSD without overwhelming the existing grammar and heuristics. Augmenting the constituent's score by a small factor every round decreases but does not eliminate the possibility of being pruned.

Progressive hints are specified as a tuple $(w, i, t, s)$, where $w$ is the base-form of a word, $i$ is an interval representing the span of the word, $t$ is a trips type and $s$ is a score representing the confidence associated with the hint. The parser stores all hints for a sentence in the advice-map at the start of parsing. As constituents are selected, the advice-map is queried for word and span. For a word/span query, $\left(w_{c}, i_{c}, t_{c}\right)$ from the constituent, if $w=w_{c}, i \cap i_{c}$ and $t \in \operatorname{ancestors}\left(t_{c}\right)$, we augment the constituent score by $s$. Specifically,

$$
\operatorname{augment}\left(s_{c}, s, \alpha\right)=\alpha *\left(s_{c} * s\right)+(1-\alpha), 0 \leq \alpha \leq 1
$$

The final score is computed recursively as the average of the augment of the root constituent and the score of its children.

\section{Semantic Factor Agreement}

We produce a factorized version of the ontology by partitioning the TRIPS ontology at nodes which have a semantic structure different to their parent. The resulting partition has the property that all members of a partition have the same role structure and restrictions and could therefore be substituted with each other for a structurally valid parse. It follows that for two nodes to fall in different partitions, they have a specific, quantifiable difference in meaning which can directly impact parse structure. We apply this concept to determine the severity of sense deviances. For a word instance, if the predicted sense falls in the same semantic factor as the gold tag, the predicted sense will not cause a significant structural change to the rest of the parse.

\section{Evaluation}

We report three metrics to evaluate the performance of the semantic parser - (i) accuracy (exact sense agreement) (ii) Mean Wu-Palmer similarity (Wu and Palmer 1994) and (iii) Mean SemFac (computed as Wu-Palmer over the factorized ontology described above). We find that sense decisions provided by SupWSD are able to substantially improve the decisions made by the TRIPS parser. Correct decisions from SupWSD improve the general accuracy of the parser while incorrect decisions from SupWSD do not cause cascading errors.

Table 1 shows that the plain parser significantly underperforms compared to SupWSD. However, we see improvements across all metrics in both the pre and prog strategies of hinting the parser. When the strategies are combined we see a significant improvement across all metrics, with roughly $14 \%$ improvement to raw accuracy.

\section{Future Work and Conclusion}

In this work, we use SupWSD, a statistical WSD system, to advise the TRIPS parser, a logical semantic parser. Our initial attempts at providing advice both to initialize and guide the parser elicit significant improvements in the word sense decisions output by the parser. We define and use SemFac similarity over the TRIPS ontology to examine impact of modified senses in the parser output and determine that the resulting senses do not introduce significant new conflicts in semantic restrictions. We find that introducing word sense advice can elicit greater improvements in the quality of semantic parsing without introducing significant new errors.

Future work will involve building richer advice models for the parser. Additional hints can be provided during prehinting to score the likelihood of senses entering the chart. Additional progressive hinters can utilize tags from other systems such as semantic role labelers or named entity recognizers. WSD models trained directly on TRIPS ontology types can also have significant benefits.

\section{References}

Allen, J. F.; Swift, M.; and De Beaumont, W. 2008. Deep semantic analysis of text. In Proceedings of the 2008 Conference on Semantics in Text Processing, 343-354. Association for Computational Linguistics.

Fellbaum, C. 1998. A semantic network of english verbs. WordNet: An electronic lexical database 3:153-178.

Navigli, R.; Jurgens, D.; and Vannella, D. 2013. Semeval2013 task 12: Multilingual word sense disambiguation. In Second Joint Conference on Lexical and Computational Semantics (* SEM), Volume 2: Proceedings of the Seventh International Workshop on Semantic Evaluation (SemEval 2013), volume 2, 222-231.

Papandrea, S.; Raganato, A.; and Bovi, C. D. 2017. Supwsd: A flexible toolkit for supervised word sense disambiguation. In Proceedings of the 2017 Conference on Empirical Methods in Natural Language Processing: System Demonstrations, 103-108.

Wu, Z., and Palmer, M. 1994. Verbs semantics and lexical selection. In Proceedings of the 32nd annual meeting on Association for Computational Linguistics, 133-138. Association for Computational Linguistics. 